\title{
Análise das Infraestruturas do Censo Escolar 2011: uma proposta da disciplina de Tópicos em Banco de Dados
}

\author{
Marcelo S. Santos, Daniela B. Claro, André Mario de Souza Oliveira Filho, \\ Antonio R. Paoli, Carlos D. B. Barbosa, Erick V. G. Almeida, Gustavo D. de \\ Araújo Carvalho, Helder de Jesus Santana, Lucas A. Bulcão, Lucas Lara Marotta, \\ Mariana P. U. da Silva, Marcelo Pereira Novaes \\ Universidade Federal da Bahia - Instituto de Matemática - Departamento de Ciência da \\ Computação - FORMAS - Grupo de Pesquisa FORMAS (Formalismos e Aplicações \\ Semânticas) - Laboratório de Sistemas Distribuídos (LaSiD) \\ Av. Adhemar de Barros, s/n, Ondina - Salvador - Bahia 40170-110 \\ \{marceloss, dclaro, andremario, paoli, douglasd3, erikvinicius5, gdacarv, helderjs, \\ lucasbulcao, lucasmarotta mariperdiz, marcelonovaes\}@dcc.ufba.br
}

\begin{abstract}
Resumo. A partir dos dados do Censo Escolar de 2011 disponibilizado pelo INEP, buscou-se minerar as regiões onde estas escolas estão distribuídas com o intuito de fornecer um panorama das infraestruturas das escolas no país. $O$ trabalho foi desenvolvido com os alunos da disciplina de Tópicos em Banco de Dados através da aplicação das regras de associação por região. Os alunos envolvidos neste projeto puderam analisar e comparar os seus resultados com as demais regiões, permitindo assim um crescimento mútuo em relação ao assunto e a disciplina.

Abstract. We mined the School Census 2011 data provided by INEP divided into regions. These schools were distributed in order to provide an overview of their infrastructure in our country. The study was conducted with students of the discipline of Topics in Database through the application of association rules by region. Students involved in this project were able to analyze and compare their results with other regions, thus enabling mutual growth in relation to the subject and the course.
\end{abstract}

\section{Introdução}

A análise de problemas reais da sociedade brasileira tem motivado diversas pesquisas na área de Banco de Dados. O desenvolvimento destas pesquisas associadas ao conteúdo proposto por uma disciplina, motiva o corpo discente a pesquisar novas soluções e se aprofundar no conteúdo apresentado.

A disciplina de Tópicos em Banco de Dados do curso de Ciência da Computação na Universidade Federal da Bahia (UFBA) é uma disciplina de ementa livre onde diversos temas de pesquisa da área de Banco de Dados podem ser propostos e estudados. Neste semestre de 2014.1, a proposta foi um estudo da Mineração de Dados, através da análise das regras de associação em uma base de dados real. A base de dados utilizada foi o Censo Escolar 2011 [INEP, 2011], no qual somente o último ano e as informações da infraestrutura das escolas foram utilizados devido ao grande volume disponível e por corresponder aos dados mais atuais. 
De acordo com alguns estudos, a infraestrutura das escolas tem impacto na qualidade do ensino das escolas. Autores em (Castro \& Fletcher, 1986) discutem a eficiência e a eficácia do investimento na educação por parte do governo e o impacto da infraestrutura das escolas na qualidade do ensino que implica diretamente no aprendizado dos alunos. Autores em (Lee, et al., 2004) também afirmam que uma melhor infraestrutura tem impactos positivos no aprendizado dos alunos.

Diante destas pesquisas, observa-se a importância das escolas brasileiras possuírem uma infraestrutura de qualidade. Porém, um estudo realizado nos dados do censo escolar de 2011 (NETO et al. 2013) revelou que somente 0,6\% das escolas brasileiras possuíam infraestrutura considerada ideal para ensino. Este estudo também apontou que $44 \%$ das escolas básicas apresentaram uma estrutura considerada elementar. Ainda nesta pesquisa realizada em (NETO et al. 2013), os autores revelam também uma grande desigualdade regional na infraestrutura das escolas brasileiras.

O presente trabalho, diferentemente dos propostos acima, propôs analisar a associação entre os diversos atributos que compõem a base de dados do Censo Escolar 2011 com o intuito de avaliar quais as possíveis associações que podem existir em relação aos dados desta base que podem estar impactando na infraestrutura destas escolas. A pesquisa foi realizada por região, com o intuito de melhor contrapor as associações coletadas e melhor analisar os resultados.

O presente artigo está assim estruturado: a seção 2 descreve a metodologia do trabalho. A seção 3 apresenta a fundamentação teórica. A seção 4 apresenta o experimento realizado, na seção 5 discute os resultados encontrados. A seção 6 conclui o trabalho e apresenta perspectivas futuras.

\section{Metodologia}

O presente trabalho foi desenvolvido no contexto da disciplina Tópicos em Banco de Dados, onde o objetivo foi estudar as regras de associação da Mineração de Dados em uma base de dados real. A base de dados utilizada foi a Censo Escolar 2011 [INEP, 2011] com o intuito de analisar os atributos e averiguar as associações que pudessem ser obtidas a fim de melhor determinar investimentos para estas escolas por região do país.

A metodologia utilizada neste trabalho foi dividida em três etapas. A primeira etapa foi uma fase de conhecimento genérica, onde os algoritmos da Mineração de Dados foram estudados. Concomitantemente a Base de Dados Censo Escolar 2011 também foi analisada a fim de definir qual dos algoritmos seriam utilizados de acordo com os dados presentes nesta base. Para esta etapa, os alunos foram divididos em equipes e cada equipe fez um estudo e análise de um algoritmo. A segunda etapa correspondeu a definição e aplicação do algoritmo escolhido por todos os estudantes por região. Nesta etapa as regras de associação foram obtidas por equipe, consequentemente por região. A terceira etapa correspondeu a uma análise dos resultados obtidos por região, onde cada equipe expos os resultados obtidos. Estes resultados foram discutidos em diversas aulas a fim de que regras relevantes para o trabalho fossem avaliadas e encontradas.

\subsection{Descoberta do Conhecimento em Bases de Dados}

A mineração de dados é uma das etapas do processo do KDD (Knowledge Data Discovery). O (KDD) tem por objetivo extrair conhecimento de alto nível partindo de bases de dados através de métodos baseados na estatística e no aprendizado de máquina. 
O KDD é composto essencialmente de três etapas: pré-processamento, mineração de dados e pós-processamento (Tan, Steinbach, \& kumar, 2009). O pré-processamento refere-se ao tratamento do dados de entrada, onde os mesmos são transformados, selecionados e limpos. A mineração de dados consta de diversos algoritmos que serão aplicados nos dados pré-processados para obter resultados importantes.

Neste trabalho, utilizou-se as regras de associação. Conceitualmente, a regra de associação busca identificar o quanto a presença de um conjunto de itens implica na presença de algum outro conjunto distinto de itens no mesmo registro (Agrawal \& Srikant, 1994). Por fim o pós-processamento, serve para analisar os resultados obtidos.

\section{Experimentos utilizando as Regras de Associação}

O Censo Escolar é um levantamento de dados estatístico-educacionais de âmbito nacional realizado todos os anos e coordenado pelo INEP. Ele é realizado com a colaboração das secretarias estaduais e municipais de Educação e com a participação de todas as escolas públicas e privadas do país (INEP, 2011).

Antes de aplicar o algoritmo da mineração de dados, foi necessário tratar os dados das regiões para adequação ao algoritmo e busca da melhor solução.

Primeiramente foi eliminado os atributos com dados nulos, duplicados ou considerados sem relevância. A validação da relevância dos atributos foi realizada por reuniões ao longo do processo de mineração. Nesta etapa também foram removidos atributos com dados desproporcionais as suas ocorrências. Depois, os dados contínuos foram categorizados em faixas de intervalo e todos os atributos numéricos foram transformados em nominal, já que as regras de associação trabalham apenas com esse tipo de dado. Por fim, a seleção dos atributos para serem aplicados ao algoritmo foi a tarefa mais custosa, pois a base é composta por aproximadamente 120 atributos.

Após o pré-processamento dos dados, as regiões foram analisadas separadamente e seus atributos foram selecionados de maneira isolada de acordo com as características de cada região. Neste sentido, foram desenvolvidos três experimentos principais: o primeiro experimento correspondeu a utilização de todos os atributos da base de dados. No segundo experimento, cada equipe escolheu alguns atributos a serem removidos da base a fim de melhor refletir a região que estavam analisando. $\mathrm{O}$ terceiro experimento utilizou algumas análises do suporte e confiança a fim de determinar um conjunto de regras relevantes. Nos três experimentos, as equipes utilizaram a ferramenta WEKA [Hall et al., 2009], especificamente o algoritmo Apriori (Agrawal \&Srikant, 1994), a fim de gerar as regras de associação referente aos atributos selecionados.

\section{Resultados e Discussões}

Os resultados obtidos foram separados por região e consequentemente as suas discussões.

\subsection{Região Norte/Nordeste}

$\mathrm{Na}$ região Norte/Nordeste, várias regras foram geradas, das quais as seguintes foram destacadas devido a sua relevância:

1. Em 97\% dos casos onde existem alimentação nas escolas, estas não são indígenas;

2. $95 \%$ das escolas que não possuem nenhum computador por aluno, também não possuem quadra de esporte e parque infantil; 
3. $97 \%$ das escolas que possuem poucos computadores, não existe nenhum laboratório de ciência;

4. $99 \%$ das escolas que não possuem biblioteca nem parque infantil, também não possuem quadra de esporte;

5. $90 \%$ das escolas que não possuem laboratório de informática, também não possuem biblioteca nem sala de leitura;

Estas regras sinalizam que a maioria das escolas presentes nas regiões Norte e Nordeste do Brasil apresentam uma infraestrutura muito precária e, portanto, o Brasil está longe de garantir não só a equidade, mas também um padrão mínimo de qualidade na infraestrutura para o bom funcionamento dessas escolas.

Nesse sentido, fica evidente a necessidade de uma melhor distribuição de recursos relacionados a infraestrutura nessas regiões, bem como a criação de políticas públicas que possam garantir boas condições estruturais para que os alunos tenham ambientes mais favoráveis ao aprendizado.

\subsection{Região Sul}

$\mathrm{Na} \mathrm{Sul,} \mathrm{várias} \mathrm{regras} \mathrm{foram} \mathrm{geradas,} \mathrm{das} \mathrm{quais} \mathrm{as} \mathrm{seguintes} \mathrm{foram} \mathrm{destacadas} \mathrm{devido} \mathrm{a}$ sua relevância:

1. $100 \%$ das escolas em funcionamento possuem energia elétrica;

2. $100 \%$ das escolas em funcionamento possuem água;

3. $100 \%$ das escolas em funcionamento possuem esgoto;

4. $96 \%$ das escolas em funcionamento possuem cozinha;

5. $96 \%$ das escolas em funcionamento possuem TV;

Essas regras expressam que, para a região sul, todas as escolas em funcionamento possuem energia elétrica, água e esgoto. Mostra também a maioria das escolas em funcionamento possuem cozinha e TV. Isso mostra que as escolas possuem uma infraestrutura básica para o funcionamento.

\subsection{Região Sudeste}

Na região Sudeste, várias regras foram geradas, das quais as seguintes foram destacadas devido a sua relevância:

1. $100 \%$ das escolas que possuem internet, possuem computadores;

2. $93 \%$ das escolas que não possuem sala de leitura, também não possuem laboratório de ciências;

3. 93\% das escolas que possuem computadores, também possuem DVD e impressora;

4. Das escolas de São Paulo, 75\% delas possuem internet.

A partir destas regras, pode-se verificar que esta região apresenta uma boa infraestrutura em relação a tecnologia.

\subsection{Região Centro-Oeste}

$\mathrm{Na}$ região Centro-Oeste, várias regras foram geradas, das quais as seguintes foram destacadas devido a sua relevância:

1. $90 \%$ das escolas que tem diretoria e laboratório de informática estão localizadas na área urbana;

2. $81 \%$ das escolas da área urbana possuem cozinha; 
3. Mais de $57 \%$ das escolas municipais não possuem laboratório de informática, biblioteca nem quadra de esportes;

4. Mais de 55\% das escolas de Goiás não possuem laboratório de informática;

\section{Conclusão e Trabalhos Futuros}

Neste trabalho, buscou-se observar associações entre os itens da infraestrutura das escolas nas regiões do Brasil através de regras de associação da mineração de dados. A partir dos resultados obtidos, melhores investimentos podem ser realizados em cada região dependendo das necessidades observadas neste trabalho.

Diante dos resultados obtidos, verificou-se que as escolas da região do Nordeste apresentam uma infraestrutura precária, onde a maioria das escolas não possuem uma biblioteca, sala de leitura, quadra de esportes, sala de informática etc. Escolas da região Sudeste apresentam uma boa infraestrutura tecnológica, já na região Sul, a maior parte das escolas apresentam a estrutura básica para funcionamento, mas não possuem laboratório de ciências nem sala de leituras.

$\mathrm{Na}$ perspectiva dos discentes da disciplina, o trabalho foi enriquecedor pois permitiu um aprofundamento na área de Mineração de Dados, especificamente algoritmos de associação e também um contato com uma base de dados real. Além disso, o propósito do desenvolvimento deste artigo como forma de apresentação dos resultados, motivou os discentes a obterem resultados relevantes e organizarem seus resultados a fim de que pudéssemos contribuir para políticas publicas setoriais no país.

Em trabalhos futuros, pretende-se utilizar medidas de interesse das regras de associação (lift, conviction, confidence) para reduzir o tempo de análise, bem como outros algoritmos de associação (fp-growth, partition, GSP) para comparação.

\section{Referências}

Agrawal, R., \& Srikant, R. (1994). "Fast algorithms for mining association rules" In Proc. 20th int. conf. very large data bases, VLDB (Vol. 1215, pp. 487-499).

Neto, J. J. S., Jesus, G. R. D., Karino, C. A., and Andrade, D. F. D. (2013). "Uma escala para medir a infraestrutura escolar". Est. Aval. Educ., São Paulo, 24(54):78-99.

Tan, Pang-Ning, Michael Steinbach, and Vipin Kumar (2009). "Introdução ao datamining: mineração de dados". CiênciaModerna.

INEP (Instituto Nacional de Estudos e Pesquisas Educacionais Anísio Teixeira). (2011) "Censo Escolar", Disponível em: http://portal.inep.gov.br/web/educacenso/censoescolar. Último acesso em 15/07/2014

CASTRO, Cláudio de Moura; FLETCHER, Philip. (1996) “A escola que os brasileiros frequentaram em 1985”. Rio de Janeiro: Ipea, Iplan,1986.

Lee, Valerie E., Creso Franco, and Angela Albernaz. (2004) "Quality and equality in brazilian secondary schools: a multilevel cross-national school effects study." Annual Meeting of the American Educational Research Association, San Diego.

Hall, M., Frank, E., Holmes, G., Pfahringer, B., Reutemann, P., \& Witten, I. H. (2009). The WEKA data mining software: an update. ACM SIGKDD explorations newslett. 